\title{
Relación del tamaño tumoral y extensión extratiroidea con la recaída local en el cáncer diferenciado de tiroides en población mexicana
}

\author{
Javier López-Gómez ${ }^{1 *}$, Martín Granados-Garcia ${ }^{1}$, E. Alejandra Palafox-Parrilla², José Granel/ y \\ Raimundo Gutiérrez-Fonseca ${ }^{3}$ \\ ${ }^{1}$ Departamento de Cabeza y Cuello, Instituto Nacional de Cancerología, Ciudad de México, México; ${ }^{2}$ Departamento de Epidemiología, Coordinación \\ Nacional de Programas de Prevención y Control del Cáncer, Instituto Nacional de Cancerología, Ciudad de México, México; ${ }^{3}$ Servicio de \\ Otorrinolaringología, Hospital Universitario Rey Juan Carlos, Madrid, España
}

\begin{abstract}
Resumen
Introducción: El cáncer de tiroides es la segunda neoplasia con mayor incidencia en las mujeres mexicanas. Su baja mortalidad indica que podría tratarse con lobectomía sin sacrificar curación, nuestro objetivo fue encontrar un tamaño tumoral asociado con bajo riesgo de recaída local para recomendarla. Material y métodos: Se estudió una cohorte retrospectiva de 278 casos y seleccionamos 91 pacientes para un análisis. Catorce casos fueron sometidos a lobectomía y se compararon con 77 controles tratados con tiroidectomía total, previamente pareado por los principales factores pronósticos. Se estudió la relación del tamaño tumoral con la extensión extratiroidea, así mismo, el tamaño tumoral y el tipo de cirugía con la recaída. Resultados: El tamaño tumoral mayor de $2 \mathrm{~cm}$ se asoció con un RR de 5.5 (IC 95\%: 2.8-10.9) de presentar extensión extratiroidea macroscópica. Se encontró segundos primarios en el 19\% de la cohorte. Conclusiones: Existe una relación entre el tamaño tumoral y el riesgo de extensión extratiroidea. Los pacientes con tumores menores de $2 \mathrm{~cm}$ tratados con lobectomía no tuvieron riesgo de recaída distinto respecto los tratados con tiroidectomía total. La lobectomía puede realizarse en un grupo seleccionado de pacientes con tumores menores de $2 \mathrm{~cm}$.
\end{abstract}

Palabras clave: Lobectomía. Extensión extratiroidea. Cáncer diferenciado de tiroides. Recaída local. Tamaño tumoral.

\section{Association of tumor size and extrathyroid extension with local relapse in differentiated thyroid cancer in the Mexican population}

\begin{abstract}
Introduction: Thyroid cancer is the second neoplasm with higher incidence in Mexican women. Its low mortality indicates that it could be treated with hemithyroidectomy without sacrificing cure, our objective was to find a tumor size associated with a risk of local relapse to recommend this procedure. Material and methods: We studied a retrospective cohort of 278 cases, and selected 91 patients for a matched pair analysis. There were 14 cases treated with hemithyroidectomy alone and they were compared with 77 controls treated with total thyroidectomy and previously paired for the main prognostic factors. The relationship between tumor size and extrathyroid extension was studied. Also the tumor size and the extension of the surgery with relapse. Results: Tumor size greater than $2 \mathrm{~cm}$ was associated with a RR 5.5 of presenting extrathyroidal macroscópica extension, $19 \%$ of patients had second malignancies. Conclusions: There is a relationship between extrathy-
\end{abstract}

Correspondencia:

*Javier López-Gómez

E-mail: dr.javierlopgom@gmail.com
Disponible en internet: 11-11-2019 Gac Mex Oncol. 2020;19(1):7-14 www.gamo-smeo.com (http://creativecommons.org/licenses/by-nc-nd/4.0/). 
roidal extension and tumor size. Patients with tumor size less than $2 \mathrm{~cm}$ and treated with hemithyroidectomy alone were not distinct in relapse rate when compared with those treated with total thyroidectomy. Therefore, hemithyroidectomy can be appropriate for selected patients with tumors smaller than $2 \mathrm{~cm}$.

Key words: Lobectomy. Extrathyroidal extension. Differentiated thyroid cancer. Local recurrence. Tumor size.

\section{Introducción}

Existe controversia relativa al mejor manejo quirúrgico del carcinoma diferenciado de tiroides. Algunos apoyan la tiroidectomía total sistemática, ya que facilitaría la ablación con yodo y la detección de recaída o persistencia, además, en centros con experiencia la morbilidad resulta ser mínima; sin embargo, otros favorecen la lobectomía en casos seleccionados para reducir la morbilidad asociada a la tiroidectomía total, como el hipoparatiroidismo permanente y disminuir en un gran porcentaje la administración de hormonas tiroideas, aunque se ha documentado mayor riesgo de recaída en el lóbulo tiroideo remanente $(14 \%)$ debido a la frecuente multicentricidad del carcinoma papilar, que alcanza el $40 \%$. Sin embargo, se ha documentado que con una correcta selección de pacientes la tasa de recaída puede ser tan baja como del 1 al $4 \%$ y la tasa de tiroidectomías complementarias menor del $10 \%$, sin afectar la supervivencia ${ }^{1-6,12}$.

El tamaño tumoral con el cual se recomienda la tiroidectomía total ha sido motivo de controversia, con un rango de 1 a $4 \mathrm{~cm}$, sin embargo, en las principales guías de tratamiento se recomienda practicar tiroidectomía total en pacientes con tumores mayores de $4 \mathrm{~cm}$, con extensión extratiroidea macroscópica o ante la evidencia de enfermedad regional o a distancia ${ }^{8}$, aunque previamente se había propuesto un tamaño de corte de 1 $\mathrm{cm}$. Esto refleja lo poco que se ha estudiado este punto. Creemos que el tamaño tumoral creciente se asocia con un mayor riesgo de extensión extratiroidea macroscópica. Esto es importante, porque determina un riesgo de recaída local que expone la supervivencia del paciente. Por otra parte esto es distinto de la recaída local en el lóbulo contralateral cuando se practica lobectomía, y que regularmente es rescatada con nueva cirugía sin impactar en la supervivencia a largo plazo. Creemos que el tamaño tumoral no ha sido suficientemente analizado como indicador de la extensión adecuada de la cirugía, por ello emprendimos este estudio.

\section{Objetivo}

Encontrar qué tamaño tumoral está asociado con un bajo riesgo de extensión extratiroidea macroscópica para recomendar la lobectomía tiroidea.

\section{Metodología}

Se obtuvo la autorización del Comité de ética e investigación del Instituto Nacional de Cancerología (INCan) con folio 0060 y revisamos los registros de los pacientes con nuevo diagnóstico de cáncer diferenciado de tiroides ingresados al INCan de septiembre del 2006 a septiembre del 2016. Se identificaron 749 casos, pero incluimos en la cohorte retrospectiva solo a los pacientes tratados completamente en el INCan, con diagnóstico corroborado de carcinoma papilar o carcinoma folicular y registros completos. Se excluyeron los pacientes con diagnóstico distinto, tratados fuera del INCan o con registros incompletos. Persistieron 278 pacientes para el análisis.

Analizamos las siguientes variables independientes: edad, sexo, tamaño tumoral $(\mathrm{T})$, estado ganglionar $(\mathrm{N})$, presencia de metástasis $(\mathrm{M})$, variantes histológicas, multicentricidad, diseminación perineural, permeación linfovascular, seguimiento, extensión de cirugía, etapa clínica de acuerdo con el American Joint Committe on Cancer (AJCC) 2018 y grupo de riesgo según la American Thyroid Association (ATA) 2015, antecedentes de radioterapia a cuello, antecedentes familiares de cáncer de tiroides y presencia de otros tumores primarios. Y como variables dependientes la extensión extratiroidea y la recaída.

La extensión extratiroidea se clasificó como: a) ausente, cuando el tumor se limitó a la cápsula, sin extensión; b) microscópica, cuando el tumor no respetó la cápsula e invadía la grasa peritiroidea, pero respetaba estructuras adyacentes, y c) macroscópica o gruesa, cuando el tumor no respetaba la cápsula y se extendía a estructuras adyacentes (músculo, nervio laríngeo recurrente, tráquea, laringe, esófago, faringe, vasos sanguíneos o tejidos blandos).

La recaída se clasificó de acuerdo con la ATA (2015) en: a) recaída bioquímica, si tiroglobulina $(\mathrm{Tg})$ superior a $0.2 \mathrm{ng} / \mathrm{ml}$ con hormona estimulante de la tiroides (TSH) suprimida, o arriba de $1 \mathrm{ng} / \mathrm{ml}$ con TSH estimulada y en ausencia de anticuerpos anti-Tg; b) recaída local, en presencia clínica o por imagen de tumor macroscópico en el lecho tumoral, corroborado por biopsia por aspiración con aguja fina (BAAF) o documentado en el reporte final de patología; c) recaída regional, en 


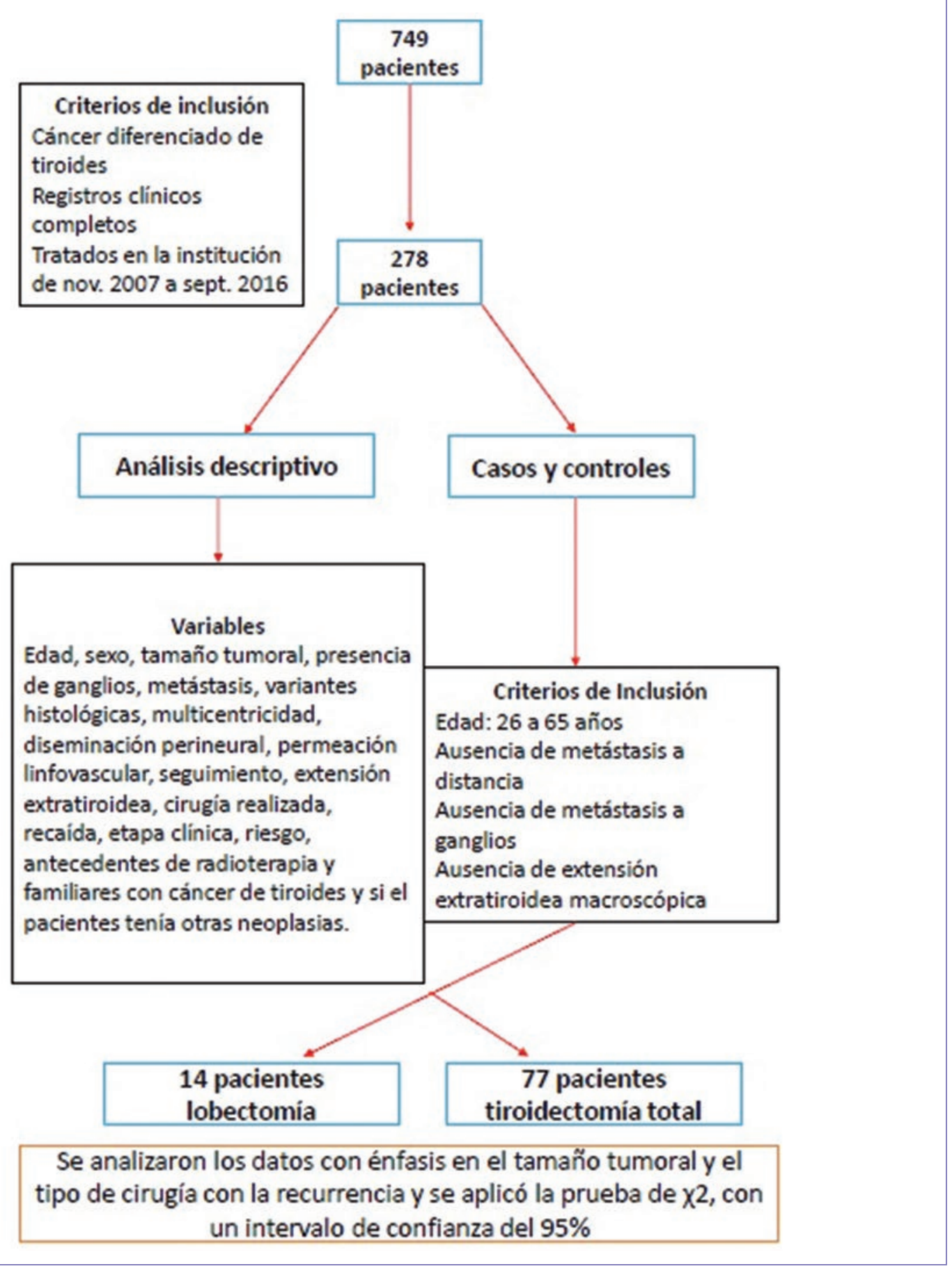

Figura 1. Algoritmo de metodología.

presencia clínica o por imagen de tumor macroscópico en las cadenas ganglionares y corroborado por BAAF o en el reporte final de patología, y d) recaída a distancia, con evidencia de actividad metastásica por tomografía axial computarizada, tomografía por emisión de positrones (PET/CT) o rastreo con iodo después de 4 meses del tratamiento. Se analizaron las diferentes combinaciones de recaída.
De 278 pacientes que inicialmente cumplieron los criterios de inclusión, se realizó un estudio de casos y controles anidado. El cálculo del tamaño de muestra fue por conveniencia y se incluyeron todos los casos de lobectomía, 14 casos sometidos a lobectomía y 77 controles tratados con tiroidectomía total y que fueron pareados por los principales factores pronósticos de acuerdo con la AJCC 2018 y ATA 2015 para controlar 
Tabla 1. Tipo de recurrencia y recurrencia agrupada

\begin{tabular}{|l|c|c|c|}
\hline \multicolumn{2}{|l|}{ Tipo de recurrencia Recurrencia agrupada } & \\
\hline Tipo de recurrencia & $12(4.31 \%)$ & & $12(4.31 \%)$ \\
\hline Bioquímica & $30(10.79 \%)$ & & $10(3.56 \%)$ \\
\hline Distancia & $4(1.43 \%)$ & Bioquímica & $57(20.50 \%)$ \\
\hline Local & $3(1.07 \%)$ & Local (todos los tipos) & \\
\hline Local y distancia & $1(0.35 \%)$ & Otros (regional y distancia) & \\
\hline Local y regional & $2(0.71 \%)$ & & \\
\hline Local, distancia y regional & $16(5.75 \%)$ & & \\
\hline Regional & $11(3.95 \%)$ & & \\
\hline Regional y distancia & $199(71.58 \%)$ & \\
\hline Sin recurrencia & & \\
\hline
\end{tabular}

los factores de confusión con la recaída (estos son la edad, ausencia de metástasis ganglionares o a distancia y ausencia de extensión extratiroidea macroscópica obvia en el preoperatorio o transoperatorio).

Se analizaron los datos, se midió la asociación entre el tamaño tumoral y la extensión extratiroidea con riesgo relativo y a la vez el tamaño tumoral y la extensión de cirugía con el riesgo de recaída aplicando la prueba de $\chi^{2}$, con un intervalo de confianza del 95\% (Fig. 1). La significancia estadística se asumió con un valor de p menor de 0.05 .

\section{Resultados}

Se identificaron 278 pacientes: 38 hombres (13.67\%) y 240 mujeres (86.33\%), en una proporción 6:1. El promedio de seguimiento fue de 60 meses, con rango de 300 a 3,751 días. Mayores de 55 años fueron 66 pacientes (23.74\%) y menores de 55 años 212 (76.26\%). Con 5 años de seguimiento en promedio, 199 (71.58\%) estaban libres de enfermedad y 79 (28.42\%) tenían persistencia o recaída. De las recaídas, el $15.18 \%$ fue bioquímica, el $12.65 \%$ estructural local y el $72.15 \%$ estructural regional o a distancia. Solo dos habían muerto con actividad tumoral; al ser un tumor con baja mortalidad no se realizó análisis de supervivencia global (Tabla 1).

Se catalogaron como de riesgo bajo de acuerdo con la ATA (2015) 36 pacientes (12.95\%), en riesgo intermedio $106(38.13 \%)$ y como alto riesgo 136 pacientes (48.92\%). La recaída en relación con grupos de riesgo mostró entre los pacientes con alto riesgo una recaída del $36.76 \%$, de ellos la recaída bioquímica representó el $6.61 \%$, la local el $3.67 \%$ y distancia o regional el $26.47 \%$. En riesgo intermedio la recaída fue del $24.52 \%$, bioquímica en el $2.83 \%$, local en el $3.77 \%$ y regional o a distancia en el $17.92 \%$. En bajo riesgo la recaída fue del $8.33 \%$; sin recaídas bioquímicas, pero recaída local del $2.77 \%$ y regional o a distancia del $5.55 \%$. Dicho de otra forma, la ausencia de enfermedad (AE) en bajo riesgo fue del $91.66 \%$, en riesgo intermedio del $75.47 \%$ y en alto riesgo del $63.2 \%$. Según la AJCC 2018 se encontraron en etapa I 212 pacientes (76.26\%), en etapa II 56 pacientes $(20.14 \%)$, en etapa III $4(1.44 \%)$ y en etapa metástasica $6(2.16 \%)$.

A criterio del médico tratante fueron tratados con lobectomía 15 pacientes (5.39\%), con tiroidectomía total $151(54.31 \%)$, con tiroidectomía total más disección de cuello $105(37.76 \%)$ y tiroidectomía total extendida 7 pacientes (2.51\%); este último grupo representa el cáncer localmente avanzado con invasión a estructuras adyacentes.

La histología más común fue cáncer papilar convencional en 202 (72.66\%), 49 (17.62\%) tenían carcinoma papilar con alguna variante de mal pronóstico, 16 (5.75\%) tenían microcarcinoma y en 11 (3.95\%) se diagnosticó carcinoma folicular.

Doce pacientes (4.3\%) tenían antecedentes heredofamiliares de cáncer de tiroides y $15(5.42 \%)$ antecedente de radioterapia a cuello, además 53 pacientes (19.06\%) tenían segundos tumores primarios; de ellos, el cáncer de mama representó el 37.03\%, el cáncer 
Tabla 2. Tamaño tumoral y extensión extratiroidea

\begin{tabular}{|c|c|c|c|c|c|}
\hline Tamaño tumoral & 0 a $1 \mathrm{~cm}$ & 1 a $2 \mathrm{~cm}$ & 2 a $4 \mathrm{~cm}$ & Mayor de $4 \mathrm{~cm}$ & Total \\
\hline Sin extensión/microscópica & $\begin{array}{c}38 \\
(97.4 \%)\end{array}$ & $\begin{array}{c}74 \\
(87.1 \%)\end{array}$ & $60(64.5 \%)$ & $\begin{array}{c}36 \\
(59 \%)\end{array}$ & $208(74.8 \%)$ \\
\hline Extensión macroscópica & $\begin{array}{c}1 \\
(2.6 \%)\end{array}$ & $\begin{array}{c}11 \\
(12.9 \%)\end{array}$ & $33(35.5 \%)$ & $\begin{array}{c}25 \\
(41 \%)\end{array}$ & $70(25.2 \%)$ \\
\hline Total & $\begin{array}{c}39 \\
(14 \%)\end{array}$ & $\begin{array}{c}85 \\
(30.6 \%)\end{array}$ & $\begin{array}{c}93 \\
(33.5 \%)\end{array}$ & $\begin{array}{c}61 \\
(21.9 \%)\end{array}$ & 278 \\
\hline
\end{tabular}

Pearson $\chi^{2}=30.674$

$\mathrm{p}=0.000$.

cervicouterino el $13.2 \%$, el linfoma no Hodgkin el $7.54 \%$, el cáncer de piel no melanoma y el cáncer de endometrio el $5.66 \%$ y el linfoma folicular y el sarcoma retroperitoneal un $3.77 \%$ cada uno. Además, ocurrió en un $1.88 \%$ en los siguientes cánceres: renal, de parótida, tipo linfoma de tejido linfoide asociado a mucosas (MALT), de cavidad oral, próstata, ovario, sarcoma pulmonar, leucemia, tumor maligno de la vaina periférica, melanoma, esófago y linfoma linfoblástico.

Entre 278, 52 pacientes no mostraron extensión extratiroidea (18.71\%), 155 (55.76\%) tenían extensión microscópica y $71(25.54 \%)$ extensión macroscópica; 39 pacientes tenían tumores de 0 a $1 \mathrm{~cm}(14.02 \%), 84$ de 1 a $2 \mathrm{~cm}(30.21 \%), 94$ pacientes de 2 a $4 \mathrm{~cm}$ (33.81\%) y 61 pacientes tenían tumores mayores de $4 \mathrm{~cm}$ (21.94\%).

Cuando se estudió la extensión extratiroidea macroscópica con el tamaño tumoral se encontró que el riesgo de extensión extratiroidea macroscópica se incrementó significativamente a partir del grupo de 2 a $4 \mathrm{~cm}$, con 33 pacientes (35.5\%), seguido de $25(41 \%)$ en el grupo con tumor mayor de $4 \mathrm{~cm}$, así mismo en 11 pacientes (12.9\%) en el grupo de 1 a $2 \mathrm{~cm}$ y 1 paciente (2.6\%) en el grupo de 0 a $1 \mathrm{~cm}$.

La extensión extratiroidea microscópica se encontró en 59 pacientes $(38.06 \%)$ en el grupo de 1 a $2 \mathrm{~cm}$, en 49 (31.61\%) en el grupo de 2 a $4 \mathrm{~cm}$, en 30 (19.35\%) en el grupo de mayor de $4 \mathrm{~cm}$ y en $17(10.96 \%)$ en el grupo de 0 a $1 \mathrm{~cm}$. No se documentó extensión extratiroidea en el grupo de 0 a $1 \mathrm{~cm}$ con 21 pacientes (40.38\%), 13 (25\%) en el grupo de 1 a $2 \mathrm{~cm}, 12(23.07 \%)$ en el grupo de 2 a $4 \mathrm{~cm}$ y en 6 pacientes (11.53\%) con tumor mayor de $4 \mathrm{~cm}$.

El mayor número de pacientes con extensión extratiroidea ocurrió en el grupo de 2 a $4 \mathrm{~cm}$, con 93 pacientes (33.5\%); con más detalle, la extensión extratiroidea macroscópica ocurrió en el $2.6 \%$ en el grupo de tumores de 0 a $1 \mathrm{~cm}$, el $14.9 \%$ en el grupo de $1 \mathrm{a} 2 \mathrm{~cm}$, en el $35.5 \%$ el grupo de 2 a $4 \mathrm{~cm}$ y en el $40.1 \%$ el grupo de
Tabla 3. Estimación del riesgo

\begin{tabular}{|l|c|c|c|}
\hline \multicolumn{4}{|c|}{ Estimación del riesgo } \\
\hline & Valor & $\begin{array}{r}\text { Intervalo de confianza } \\
\text { del 95\% }\end{array}$ \\
\hline & 5.531 & 2.806 & 10.903 \\
\hline Riesgo relativo para TT & 1.442 & 1.260 & 1.650 \\
\hline Sin ETT macroscópica & 0.261 & 0.147 & 0.463 \\
\hline Con EET macroscópica & & & Superior \\
\hline
\end{tabular}

TT: tamaño tumoral; ETT: extensión extratiroidea.

Tabla 4. Cirugía y tipo de recurrencia

\begin{tabular}{|l|c|c|c|}
\hline \multicolumn{4}{|c|}{ Cirugía y tipo de recurrencia } \\
\hline Recurrencia & Lobectomía & Tiroidectomía total & Total \\
\hline Bioquímica & 0 & 1 & 1 \\
\hline Local & 0 & 2 & 2 \\
\hline $\begin{array}{l}\text { Otro tipo de } \\
\text { recurrencia }\end{array}$ & 0 & 5 & 5 \\
\hline $\begin{array}{l}\text { Sin } \\
\text { recurrencia }\end{array}$ & 14 & 69 & 83 \\
\hline Total & 14 & 77 & 91 \\
\hline
\end{tabular}

Pearson $\chi^{2}(3)=1.595$.

Exacta de Fisher $=1.184$.

$p=0.661$.

tumores mayores de $4 \mathrm{~cm}$. Se calculo un riesgo relativo de 5.5 en tumores mayores de $2 \mathrm{~cm}$ para extensión extratiroidea macroscópica, siendo estadísticamente significativo (IC 95\%: 2.8-10.9) (Tablas 2 y 3).

En las tablas 4 a 6 y en las figuras 2 y 3 mostramos el análisis limitado a los 91 pacientes pareados por los principales factores pronóstico. En ellos no hubo 
Tabla 5. Tipo de recurrencia y tamaño tumoral

\begin{tabular}{|c|c|c|c|c|}
\hline \multicolumn{5}{|c|}{ Tipo de recurrencia y tamaño tumoral } \\
\hline Recurrencia & 0 a $1 \mathrm{~cm}$ & 1 a $2 \mathrm{~cm}$ & Más de $2 \mathrm{~cm}$ & Total \\
\hline Bioquímica & 0 & 1 & 0 & 1 \\
\hline Local & 1 & 0 & 1 & 3 \\
\hline $\begin{array}{l}\text { Otro tipo de } \\
\text { recurrencia }\end{array}$ & 0 & 3 & 2 & 5 \\
\hline $\begin{array}{l}\text { Sin } \\
\text { recurrencia }\end{array}$ & 26 & 34 & 23 & 83 \\
\hline Total & 28 & 34 & 29 & 91 \\
\hline
\end{tabular}

Tabla 6. Cirugía y tamaño tumoral

\begin{tabular}{|l|c|c|c|}
\hline \multicolumn{4}{|c|}{ Cirugía y tamaño tumoral } \\
\hline Tamaño & Lobectomía & Tiroidectomía & Total \\
\hline 0 a $1 \mathrm{~cm}$ & 5 & 22 & 27 \\
\hline 1 a $2 \mathrm{~cm}$ & 7 & 31 & 38 \\
\hline Más de $2 \mathrm{~cm}$ & 2 & 24 & 26 \\
\hline Total & 14 & 77 & 91 \\
\hline
\end{tabular}

Pearson $\chi^{2}(2)=1.655$

Exacta de Fisher $=1.669$

$\mathrm{p}=0.437$.

relación entre la extensión de la cirugía con el tipo de recaída $(p=0.661)$ (Tabla 4) (Fig. 2). Tampoco entre el tipo de recaída y el tamaño del tumor $(p=0.537)$ (Tabla 5) (Fig. 3), ni entre la extensión de la cirugía con el tamaño tumoral $(p=0.437$ ) (Tabla 6$)$.

\section{Discusión}

El carcinoma diferenciado de tiroides es ahora la segunda neoplasia con mayor incidencia entre las mujeres mexicanas, luego del cáncer de mama ${ }^{13}$; pero a pesar de su creciente incidencia, la mortalidad se mantiene baja y constante, esto sugiere que existe sobretratamiento de una neoplasia relativamente indolente, por ello creemos que casos seleccionados podrían ser tratados en forma menos agresiva para evitar una morbilidad inapropiada, como el hipoparatiroidismo permanente, disminuir la necesidad de tomar hormonas tiroideas y lesiones del nervio laríngeo recurrente, sin sacrificar la probabilidad de supervivencia a largo plazo.

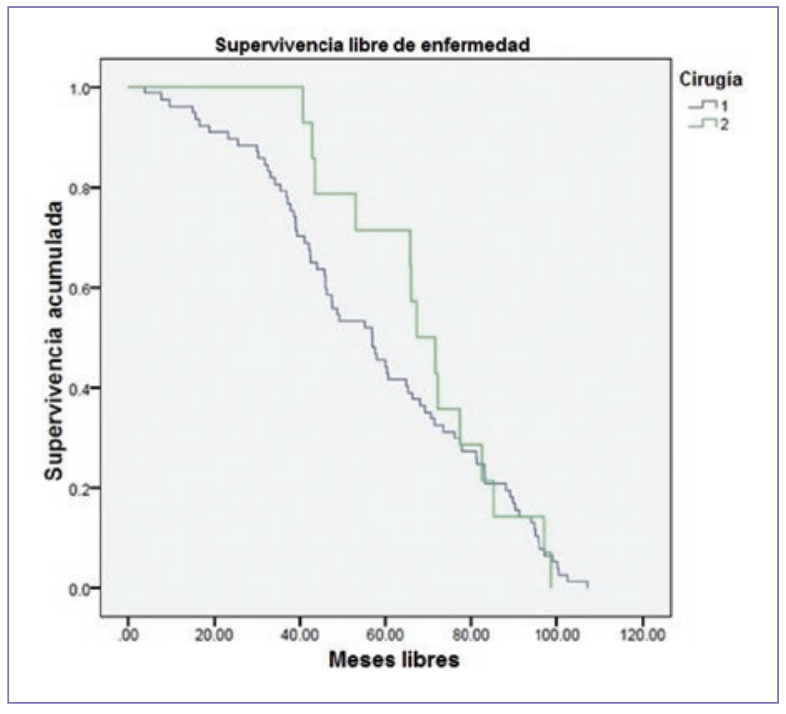

Figura 2. Media de supervivencia libre de recurrencia (cirugía - recurrencia). Cirugía: 1) tiroidectomía total 57.97 meses; 2) lobectomía 68.81 meses.



Figura 3. Media de supervivencia libre de recurrencia (tamaño de tumor - recurrencia): 0 a 1cm, 50.91 meses; 1 a $2 \mathrm{~cm}, 63.89$ meses; y más de $2 \mathrm{~cm}, 62.49$ meses.

En nuestra serie encontramos una mayor proporción de pacientes menores de 55 años de edad y mujeres respecto lo publicado por otros autores (4:1), esto representa un grupo con mejor pronóstico de supervivencia, donde un tratamiento eficaz y asociado a menor morbilidad es deseable.

Como es de esperar, encontramos que el carcinoma papilar convencional fue el tipo histológico más común, seguido de variantes del carcinoma papilar con mal 
pronóstico, microcarcinomas y, lo menos frecuente, carcinoma folicular, coincidente con lo publicado. Encontramos una elevada tasa de segundos primarios, que en su mayoría está representado por el cáncer de mama, seguido del cáncer cervicouterino y el linfoma no Hodgkin. Esto se debe a varios motivos: 1) estos pacientes suelen coincidir con los tumores más pequeños, encontrados de manera incidental mediante uso de PET/CT para estudio de sus tumores primarios; 2) la presencia de un sesgo por ser un centro de referencia oncológico, y 3) podrían representar síndromes genéticos, como el de Cowden (en el caso de mama), los cuales no se estudiaron en el departamento de genética ${ }^{13}$.

En concordancia con la literatura, entre los 278 pacientes se obtuvo una supervivencia libre de enfermedad a 5 años del $71.58 \%$, solo el $28.42 \%$ tenían persistencia o recaída, de ellos dos habían muerto con actividad tumoral. En las etapas I y II la mayor parte de nuestros pacientes están vivos sin actividad tumoral, y en la literatura tienen una supervivencia a 10 años del 97 al 100\%. Los pacientes con etapa clínica III, junto con los pacientes que requirieron una resección extendida, representan el cáncer localmente avanzado y todos tienen actividad tumoral al momento del análisis; se ha descrito en este grupo una supervivencia a 10 años del 88 a 95\%. El porcentaje de pacientes con enfermedad metastásica fue acorde a lo descrito (2 a $5 \%)^{11,14}$.

La tasa de recaída concuerda con lo reportado en la literatura, del $28.42 \%$; de ellos, la mayoría tuvo extensión extratiroidea (87\%). De las recaídas, la amplia mayoría fue regional y/o distancia, seguido de la bioquímica y la estructural local, con porcentajes muy próximos a los reportados ${ }^{3,7,8,11}$.

En cuanto al porcentaje de AE por grupos de riesgo, coincidió con lo reportado por la ATA excepto en alto riesgo, donde encontramos un porcentaje mayor de pacientes sin enfermedad de casi el doble (63.2 vs. $32 \%)$; consideramos que este hallazgo podría deberse a falta de años de seguimiento en el análisis. La recaída con enfermedad estructural en riesgo bajo es del 1 al $2 \%$ y el riesgo intermedio del $8 \%$, lo cual es comparable a lo encontrado en esta serie ${ }^{11}$.

Los pacientes con extensión extratiroidea macroscópica fueron 70 pacientes $(25.2 \%)$, la mayoría se encuentra en el grupo de 2 a $4 \mathrm{~cm}$, de ellos el $64.78 \%$ están vivos sin actividad tumoral, comparable al porcentaje de $A E$ entre los pacientes con alto riesgo. Cuando se hace una asociación de riesgo entre tamaño tumoral y la extensión extratiroidea macroscópica corroboramos que se hace significativa en tumores mayores de $2 \mathrm{~cm}$ (35\%), prácticamente duplicándose el riesgo respecto a los tumores menores de $2 \mathrm{~cm}$ (15\%), dicho de otra forma: un paciente que presenta un tumor mayor de $2 \mathrm{~cm}$ tiene 5.5 veces más riesgo de tener extensión extratiroidea macroscópica.

La optima extensión de la cirugía siempre ha sido motivo de controversia. Los que proponen la tiroidectomía total argumentan que se asocia a mejor control local y que facilita el seguimiento mediante ablación, que a su vez permite las mediciones seriadas de Tg. Otros autores sostienen que la lobectomía selectiva es tratamiento suficiente y adecuado para un grupo de pacientes con características pronósticas favorables y que la tiroidectomía total sistemática significa un sobretratamiento que expone al paciente a un riesgo innecesario de morbilidad y que no existe beneficio de administrar yodo, ya que estos pacientes tienen una supervivencia casi perfecta.

Asumiendo que un grupo de pacientes son susceptibles de tratamiento apropiado exclusivamente con lobectomía sin aumentar el riesgo de recaída ni mortalidad, las características de este grupo son controvertidas, en especial en lo relativo al tamaño tumoral. Las recomendaciones han variado, desde practicarla en todos los tumores menores de $5 \mathrm{~cm}$ hasta evitarla en todos los tumores mayores de $1 \mathrm{~cm}$, sin embargo esta recomendación nunca provino de algún estudio específico.

Teorizamos que el tamaño tumoral guarda relación con el riesgo de extensión extratiroidea y el tamaño tumoral es susceptible de medición ultrasonográfica consistente en el preoperatorio. Si esto es cierto, el tamaño tumoral elegido como nivel de corte para lobectomía debe asociarse a una baja probabilidad de extensión extratiroidea macroscópica, ya que esta se asocia a un alto riesgo de recaída local, por ello se requiere administrar tratamiento adyuvante para abatir los riesgos, y esto solo puede hacerse en forma eficaz eliminando todo el tejido tiroideo. Además, para practicar solo lobectomía se deben reunir otras condiciones, como carecer de enfermedad multicéntrica macroscópica, extensión extratiroidea macroscópica en el preoperatorio o transoperatorio, enfermedad regional 0 a distancia.

Nuestro estudio busca definir el tamaño tumoral a partir del cual existe un riesgo significativo de extensión extratiroidea macroscópica. Encontramos que a partir de los $2 \mathrm{~cm}$ existe un riesgo que supera el $35 \%$, mientras que con menores tamaños el riesgo no supera el $13 \%$, por ello creemos que solo tumores menores 
de $2 \mathrm{~cm}$ deben ser considerados para lobectomía si además reúnen las condiciones ya señaladas.

Encontramos que en pacientes seleccionados practicar lobectomía no se asocia a mayor riesgo de recaída local respecto a un grupo pareado por su principales factores pronósticos y tratado con tiroidectomía total. Si un tratamiento obtiene resultados comparables al obtenido con tiroidectomía total sin exponer al paciente a mayor morbilidad y costos, este es el mejor tratamiento.

En cuanto a las limitaciones de nuestro estudio, primero, es un estudio de cohorte retrospectivo, que introduce sesgos que intentamos controlar al parear a los pacientes usando los principales factores pronósticos. Además, tiene un número relativamente limitado de pacientes y un seguimiento promedio de 60 meses o 5 años (rango de 244 a 3,751 días), lo que podría limitar identificar eventos tardíos. Se ha descrito que el $30 \%$ de los pacientes con cáncer de tiroides desarrollarán algún tipo de recaída en el transcurso de décadas y que el $66 \%$ ocurren en la primera década, de estas, el $74 \%$ en ganglios cervicales, el $20 \%$ en el lecho quirúrgico y en la tráquea, y el $6 \%$ en músculos, de los últimos dos deriva la muerte en el $8 \%$ de los pacientes. El $21 \%$ recurrirá a distancia, siendo el pulmón el sitio más involucrado con un $63 \%$, y será la causa de muerte en el $50 \%$ de los pacientes con recaída, aunque datos actuales documentan que las recaídas ocurren en periodos más tempranos (2 a 5 años) $7,8-10$.

Nuestros resultados sugieren una relación clara entre el tamaño tumoral y la extensión extratiroidea macroscópica. Esta información abre la posibilidad de nuevos estudios. Consideramos que los pacientes con tumores menores de $2 \mathrm{~cm}$, sin metástasis (regionales y/o distancia) ni extensión extratiroidea o multicentricidad macroscópicas, pueden ser tratados con lobectomía, ya que no existe mayor riesgo de recaída. Creemos que es posible obtener resultados oncológicos similares con menor morbilidad usando lobectomía, por lo que esta debe considerarse en un grupo seleccionado de pacientes.

\section{Expresiones de gratitud}

A mi madre a la que recuerdo con amor, al Dr. Martín Granados por sus enseñanzas profesionales, personales y por su ayuda en tiempos difíciles, a la Dra. Alejandra Palafox por ayudarme a llegar a los resultados, al Instituto Nacional de Cancerología por mi formación como cirujano oncólogo y como persona. A los doctores José Granell y Raimundo Gutiérrez por su apoyo y enseñanzas en España.

\section{Bibliografía}

1. Grant CS, Hay ID, Gough IR, Bergstralh EJ, Goellner JR, McConahey WM. Local recurrence in papillary thyroid carcinoma: is extent of surgical resection important? Surgery. 1988;104:954-62.

2. Mazzaferri EL, Kloos RT. Clinical review 128: Current approaches to primary therapy for papillary and follicular thyroid cancer. J Clin Endocrinol Metab. 2001;86:1447-63.

3. Nixon IJ, Ganly I, Patel SG, Palmer FL, Whitcher MM, Tuttle RM, et al. Thyroid lobectomy for treatment of well differentiated intrathyroid malignancy. Surgery. 2012;151:571-9.

4. Vaisman F, Shaha A, Fish S, Michael Tuttle R. Initial therapy with either thyroid lobectomy or total thyroidectomy without radioactive iodine remnant ablation is associated with very low rates of structural disease recurrence in properly selected patients with differentiated thyroid cancer. Clin Endocrinol (Oxf). 2011;75:112-9.

5. Barney BM, Hitchcock YJ, Sharma P, Shrieve DC, Tward JD. Overall and cause-specific survival for patients undergoing lobectomy, near-total, or total thyroidectomy for differentiated thyroid cancer. Head Neck. 2011;33:645-9.

6. Mendelsohn AH, Elashoff DA, Abemayor E, St John MA. Surgery for papillary thyroid carcinoma: is lobectomy enough? Arch Otolaryngol Head Neck Surg. 2010;136:1055-61.

7. Mazzaferri EL, Jhiang SM. Long-term impact of initial surgical and medical therapy on papillary and follicular thyroid cancer. Am J Med. 1994; 97:418-28.

8. American Thyroid Association (ATA) Guidelines Taskforce on Thyroid Nodules and Differentiated Thyroid Cancer, Cooper DS, Doherty GM, Haugen BR, Kloos RT, Lee SL, et al. Revised American Thyroid Association management guidelines for patients with thyroid nodules and differentiated thyroid cancer. Thyroid. 2009;19:1167-214.

9. Leenhardt L, Erdogan MF, Hegedus L, et al. European thyroid association guidelines for cervical ultrasound scan and ultrasound-guided techniques in the postoperative management of patients with thyroid cancer. Eur Thyroid J. 2013;2:147-59.

10. Haugen BR, Sawka AM, Alexander EK, et al. American Thyroid Association Guidelines on the Management of Thyroid Nodules and Differentiated Thyroid Cancer Task Force Review and Recommendation on the Proposed Renaming of Encapsulated Follicular Variant Papillary Thyroid Carcinoma Without Invasion to. Thyroid. 2017;27(4):481-3.

11. National Cancer Institute 2009 [Internet]. Horner, Marie-Joseph. SEER Cancer Statistics Review 1975-2006. Disponible en: http://seer. cancer. gov/csr/1975_2006/ (2009).

12. Hay ID, Grant CS, van Heerden JA, et al. Papillary thyroid microcarcinoma: a study of 535 cases observed in a 50 -year period. Surgery. 1992;112(6):1139-46.

13. Globocan 2018. Mexico [Internet]. World Health Organization, International Agency for Research on Cancer; 2018. Disponible en: http://gco.iarc. fr/today/data/factsheets/populations/484-mexico-fact-sheets.pdf

14. Gress, Donna M, Edge, et al. Principles of cancer staging. AJCC Cancer Staging Manual. 8th ed. New York: Springer; 2017. 